hep-lat/9204006

UCLA/91/TEP/51

MCGILL/91-39

\title{
Point-Split Lattice Operators for B Decays
}

\author{
Oscar F. Hernández \\ Department of Physics \\ McGill University \\ Ernest Rutherford Physics Building \\ Montréal, Qué., Canada H3A 2T8 \\ Brian R. Hill \\ Department of Physics \\ University of California \\ Los Angeles, CA 90024
}

\begin{abstract}
The matrix element which determines the $B$ meson decay constant can be measured on the lattice using an effective field theory for heavy quarks. Various discretizations of the heavy-light bilinears which appear in this and other $B$ decay matrix elements are possible. The heavy-light bilinear currently used for the determination of the $B$ meson decay constant on the lattice suffers a substantial one-loop renormalization. In this paper, we compute the one-loop renormalizations of the discretizations in which the heavy and light fields in the bilinear are separated by one lattice spacing, and discuss their application.
\end{abstract}

12/91, to appear in Physics Letters B. 


\section{Introduction}

Several weak matrix elements involving heavy mesons can be studied on the lattice [1][2]. The relationship of the lattice operators to the operators coming from the continuum electroweak theory must be calculated in order to make use of the lattice results. While these short-distance strong interaction corrections are in principle perturbatively calculable, in practice, the one-loop corrections are sometimes so large as to be of questionable reliability. The uncertainty in whether one should use the lattice or continuum value of the strong coupling in the oneloop computation and the unknown magnitude of higher loop corrections are both measures of this uncertainty. For the operator currently in use to determine the $B$ meson decay constant on the lattice, these factors yield an uncertainty of as much as twenty per cent.

There is actually considerable freedom in the choice of the lattice operators. They are generally chosen for convenience in calculating the matrix element on the lattice and in performing the perturbative renormalization. However, the choice may also be made so as to minimize systematic effects, such as effects which vanish as a power of the lattice spacing, or the effect of perturbative corrections. For the fermion bilinear determining the decay constants of the light pseudoscalar mesons, the use of a point-split operator substantially changes the perturbative correction [3]. Similarly, it has been suggested that a bilinear suitable for the determination of the $B$ meson decay constant that is distance-one could have a smaller perturbative correction than the distance-zero operator commonly in use [4]. In this paper, we compute the renormalization of the distance-one bilinears which are suitable for determining $f_{B}$ and other $B$ decay matrix elements and which respect the symmetries of the lattice heavy quark action.

The calculation uses techniques developed and applied to calculate renormalizations of other lattice operators involving heavy quarks [5]-[7]. In the following section, we review the lattice regularization of the heavy quark effective field theory and the result for the renormalization of the operator currently being used to determine $f_{B}$. In order to concentrate on the new results and the possible applications of the new operators, we will be brief in reviewing the background for the computation and will refer the reader to the appropriate references for the technical details. Section

three contains the calculation of the one-loop renormalization of an operator with 
splitting in the spatial directions. In section four, operators with splitting in the time direction are considered. In the conclusion, we discuss the possible applications of these results.

\section{Zero-Distance Bilinears}

An expansion which analytically removes the dependence on the $b$ quark mass [8][9] can be used to determine matrix elements of $B$ mesons on the lattice [1][2]. The relationships between operators in the full theory and their counterparts in the effective theory built around the zeroth order term in this expansion [2][10]-[15] are perturbatively calculable.

Many discretizations of the heavy quark effective theory action,

$$
S=\int d^{4} x b^{\dagger}\left(i \partial_{0}+g A_{0}\right) b
$$

with the same naive continuum limit are possible. The choice of action,

$$
S=i a^{3} \sum_{n}\left[b^{\dagger}(n)\left(b(n)-U_{0}(n-\hat{0})^{\dagger} b(n-\hat{0})\right)\right]
$$

reproduces the heavy quark propagator used in numerical simulations. In the preceding two equations, $b$ is a two-component column vector which annihilates heavy quarks, $A_{0}=A_{0}^{a} T_{a}$, is the continuum $S U(3)$ gauge field, $a$ is the lattice spacing, and $U_{0}=\exp \left(i g a A_{0}\right)$ is the lattice gauge field living on time links.

The most general heavy-light bilinear in the full theory is

$$
J(x)=\bar{b}(x) \Gamma q(x)
$$

Here $\Gamma$ is any Dirac matrix, and $q$ is the light quark field. In a Dirac basis, parametrize $\Gamma$ by two-by-two blocks:

$$
\Gamma=\left(\begin{array}{ll}
\alpha & \beta \\
\gamma & \delta
\end{array}\right)
$$

In the heavy quark effective theory, the operator (2.3) takes the form

$$
b^{\dagger}(x)(\alpha \quad \beta) q(x)
$$


where $(\alpha \quad \beta)$ is a two-by-four matrix. An obvious choice of discretization of this operator which has the right continuum limit is the zero distance bilinear,

$$
b^{\dagger}(n)(\alpha \quad \beta) q(n)
$$

The light quark is treated as a Wilson fermion. Staggered and naive fermion results can be obtained by setting the Wilson mass parameter to zero [6].

In order to parametrize the relationship between the continuum operator and the lattice operator, we introduce $c$-numbers $G$ and $H$, defined by $G \Gamma=\gamma_{0} \Gamma \gamma_{0}$ and $H \Gamma=\gamma_{\mu} \Gamma \gamma_{\mu}$. We also need $H^{\prime}$, the derivative with respect to $d$ of $H$ in $d$ dimensions. This derivative depends on the extension of the gamma matrix algebra in the dimensionally regularized theory. With these definitions, the ratio of the continuum effective theory operator to the operator in the full theory is [12][16]

$$
1-\frac{g^{2}}{12 \pi^{2}}\left(C_{1} \ln \frac{m^{2}}{\mu^{2}}+C_{2}\right),
$$

where $C_{1}=5 / 2-H^{2} / 4$ and $C_{2}=-4+3 H^{2} / 4-H H^{\prime}-G H / 2$. For the case of $\Gamma=\gamma_{0} \gamma_{5}$, the bilinear used to determine $f_{B}, G=-1$ and $H=2$. If we use the extension of the gamma matrix algebra that $\gamma_{5}$ anticommutes with all the $\gamma_{\mu}, 1 \leq \mu \leq d$, then $H^{\prime}=1$. So in this case, we have $C_{1}=3 / 2$ and $C_{2}=-2$.

We also need the ratio of zero distance bilinear to the operator in the continuum effective theory. It is $[5][16]$

$$
1+\frac{g^{2}}{12 \pi^{2}}\left[d+\frac{1}{2} e+\frac{1}{2} f-\frac{5}{4}\right] .
$$

The dependence on $\mu a$ has been eliminated by setting $\mu=1 / a$. The constant

$$
d=d_{1}+d_{2} G
$$

arises from the vertex correction graphs on the lattice. When we consider the spatially split operator in the next section, we will find that its effect will be to decrease the value of $d_{1}$ and $d_{2}$. The constant $e=24.48$ arises from heavy quark wave function renormalization on the lattice. This constant should be reduced to 4.53 if one extracts $f_{B}$ from lattice results by fitting to $A \mathrm{e}^{-B n_{0} a}$ rather than $A \mathrm{e}^{-B\left(n_{0}+1\right) a}[5]$. The constant $f$ arises from light quark wave function 


\begin{tabular}{|c|c|c|c|}
\hline$r$ & $d_{1}$ & $d_{2}$ & $f$ \\
\hline 1.00 & 5.46 & -7.22 & 13.35 \\
\hline 0.75 & 5.76 & -7.23 & 11.96 \\
\hline 0.50 & 6.30 & -7.00 & 10.22 \\
\hline 0.25 & 7.37 & -5.72 & 8.07 \\
\hline 0.00 & 8.79 & 0.00 & 6.54 \\
\hline
\end{tabular}

Table 1.

renormalization on the lattice [17]. The constants $d_{1}, d_{2}$ and $f$ which depend on the Wilson mass coefficient $r$, have been calculated and tabulated previously [5][16], and analytical expressions can be found in reference [7]. For completeness, we tabulate these constants in Table 1 for several values of $r$. Errors for any of the numerically evaluated constants are at most $\mathcal{O}(1)$ in the last decimal place.

We illustrate the use of these results for $r=1.00$ and $a^{-1}=2 \mathrm{GeV}$. With $\Gamma=$ $\gamma_{0} \gamma_{5}$, the case of interest in calculating $f_{B}$, the constant $G$, which appears in equation (2.9) is -1 , as noted below equation (2.7). Taking $\mu=2 \mathrm{GeV}$ and the $b$ quark mass $m=5 \mathrm{GeV}$, with $\alpha_{S}=0.25$, the effective to full theory continuum ratio (2.7) is 0.98. The bare lattice value of $g^{2}$ at $a^{-1}=2 \mathrm{GeV}$ is approximately 1.0. For the perturbative matching however we will take $g^{2}=1.8$ which is roughly the continuum value at the scale $\pi / a[18]$. With this choice the ratio of the lattice to continuum operators is 1.31 . The value of $f_{B}$ extracted from numerical simulations from the first of the two fitting procedures mentioned above should therefore be divided by 1.28 , which is the product of these two ratios. This reduction factor would be 1.43 if the unreduced value of $e$ were used.

\section{Spatially-Split Bilinears}

A variety of other operators with the same continuum limit as the operator in equation (2.6) can be constructed. In this section, we will consider the distance-one bilinear with splitting in the spatial directions given by

$$
\frac{1}{6} \sum_{i}\left[b^{\dagger}(n+\hat{\imath}) U_{i}(n)^{\dagger}(\alpha \quad \beta) q(n)+b^{\dagger}(n-\hat{\imath}) U_{i}(n-\hat{\imath})\left(\begin{array}{ll}
\alpha & \beta
\end{array}\right) q(n)\right] .
$$

The index $i$ runs only over the three spatial directions. The sum of six terms has been chosen to respect the remnants of the $O(3)$ rotational group present in the lattice heavy quark effective theory. 
It is simplest to compute the relationship of this operator to the continuum operator (2.3) by computing its relationship to the zero-distance bilinear (2.6). Thus we will give analytical and numerical values for the change in the constants $d_{1}$ and $d_{2}$, quoted in the previous section. There are only four graphs to compute. There is the vertex correction graph which gave rise to $d_{1}$ and $d_{2}$ in the case of the zero distance bilinear, Figure 1. It is unaffected in appearance, but its value must be recomputed due to factors that arise in the spatially split case. There are also three new vertex correction graphs, which are depicted in Figure 2. Graph (a) in the figure vanishes in Feynman gauge because the gauge link at the vertex is spatial and the heavy quark field only interacts with the time component of the gauge field.

In order to quote the analytic expression for these graphs we define:

$$
\begin{aligned}
& \Delta_{1}=\sum_{\mu} \sin ^{2} \frac{l_{\mu}}{2} \\
& \Delta_{4}=\sum_{\mu} \sin ^{2} l_{\mu}, \\
& \Delta_{2}=\Delta_{4}+4 r^{2} \Delta_{1}^{2} .
\end{aligned}
$$

The sums on $\mu$ run from 1 to $4 . \Delta_{1}^{(3)}$ and $\Delta_{2}^{(3)}$ are defined to be identical to $\Delta_{1}$ and $\Delta_{2}$ respectively except with $l_{4}$ set to zero.

The Feynman integral arising from the difference of the vertex correction in the case of the spatially-split operator (3.1) and the zero-distance operator (2.6) is

$$
-\frac{4}{3} g^{2}\left(\begin{array}{ll}
\alpha & \beta
\end{array}\right) \int \frac{d^{4} l}{(2 \pi)^{4}} \frac{\left(\sum_{\sigma} \gamma_{\sigma} \sin l_{\sigma}-2 i r \Delta_{1}\right) \mathrm{e}^{i l_{0} / 2}\left(\gamma_{0} \cos l_{0} / 2+i r \sin l_{0} / 2\right) \Delta_{1}^{(3)}}{6 \Delta_{1} \Delta_{2}\left(-i\left(e^{i l_{0}}-1\right)+i \epsilon\right)}
$$

This is identical to the expression for the vertex correction for the Feynman integrand for the operator (2.6), except for an additional factor of $-2 \Delta_{1}^{(3)} / 3$. This factor necessarily vanishes for small $l$ since the two operators have the same naive continuum limit.

The second graph in Figure 2 gives

$$
\frac{4}{3} g^{2}\left(\begin{array}{ll}
\alpha & \beta
\end{array}\right) \int \frac{d^{4} l}{(2 \pi)^{4}} \frac{\left(\sum_{\sigma} \gamma_{\sigma} \sin l_{\sigma}-2 i r \Delta_{1}\right) \frac{1}{3} \sum_{i} \sin l_{i} / 2\left(\gamma_{i} \cos l_{i} / 2+i r \sin l_{i} / 2\right)}{4 \Delta_{1} \Delta_{2}}
$$

The integral for the graph in Figure 2(c), the tadpole graph, is frequently encoun- 


\begin{tabular}{|c|c|c|}
\hline$r$ & $\Delta d_{1}$ & $\Delta d_{2}$ \\
\hline 1.00 & -12.70 & 3.89 \\
\hline 0.75 & -12.98 & 4.44 \\
\hline 0.50 & -13.49 & 4.98 \\
\hline 0.25 & -14.62 & 4.81 \\
\hline 0.00 & -16.30 & 0.00 \\
\hline
\end{tabular}

Table 2. Change in $d_{1}$ and $d_{2}$ versus Wilson Mass Parameter.

tered. It is

$$
-\frac{4}{3} g^{2}(\alpha \quad \beta) \int \frac{d^{4} l}{(2 \pi)^{4}} \frac{1}{8 \Delta_{1}} .
$$

We evaluate these integrals using the techniques for isolating non-covariant poles of reference [5]. The sum of all graphs gives a change in $d_{1}$ of:

$$
\Delta d_{1}=-\frac{1}{\pi^{2}} \int d^{4} l \frac{4 \Delta_{1}-\Delta_{1}^{2}+2 \Delta_{4}+12 r^{2} \Delta_{1}^{2}}{24 \Delta_{1} \Delta_{2}}
$$

The contribution to $d_{2}$ comes from the part of the graphs that is proportional to $\gamma_{0}$. Only the graph of Figure 1 (whose Feynman integral appears in equation (3.3)) has a contribution of this type. The contribution is odd in the Wilson parameter. We find,

$$
\Delta d_{2}=\frac{r}{3} \frac{1}{\pi} \int d^{3} l \frac{\Delta_{1}^{(3)}}{\Delta_{2}^{(3)}}
$$

These expressions for $\Delta d_{1}$ and $\Delta d_{2}$ have been evaluated using VEGAS [19], and are tabulated in Table 2 for various values of the Wilson mass coefficient $r$. Errors are at most $\mathcal{O}(1)$ in the last decimal place.

As in the previous section, we illustrate the use of these results for $r=1.00$, $a^{-1}=2 \mathrm{GeV}, g^{2}=1.8$, and $\Gamma=\gamma_{0} \gamma_{5}$. The effective to full theory ratio of 0.98 remains unchanged. With $\Delta d_{1}=-12.70$ and $\Delta d_{2}=3.89, d$ is reduced to -3.91 . Then the ratio of the lattice to continuum operators is 1.06. The value of $f_{B}$ extracted from numerical simulations from the spatially-split bilinear fitting to $A \mathrm{e}^{-B n_{0} a}$ should therefore be divided by 1.04 (the product of these two ratios). As at the end of the previous section, this product would be larger, 1.18, if one fits to $A \mathrm{e}^{-B\left(n_{0}+1\right) a}$. 


\section{Temporally-Split Bilinears}

In this section, we will consider the two distance-one bilinears with splitting either forward or backward in the time direction:

$$
b^{\dagger}(n+\hat{0}) U_{0}(n)^{\dagger}(\alpha \quad \beta) q(n),
$$

and

$$
b^{\dagger}(n-\hat{0}) U_{0}(n-\hat{0})(\alpha \quad \beta) q(n) .
$$

Except for variations in the way the gauge links could be inserted between the bilinears, this exhausts the possible distance-one bilinears which respect the remnants of the $O(3)$ rotational group that is present in the lattice heavy quark effective theory. We will see that these operators do not lead to any new correlation functions on the lattice. They do however, highlight the problem of extracting decay constants in the presence of the linearly divergent heavy quark self-energy.

As in the previous section we compute the relationship of these operators to the continuum operator (2.3) by computing its relationship to the distance zero bilinear (2.6). The consideration of the new Feynman diagrams is somewhat similar to the discussion for the spatially split bilinears discussed in section three, and we will proceed directly to the contributions of the various vertex correction graphs. The graph which gave rise to $d_{1}$ and $d_{2}$ (Figure 1) must again be recomputed. However, we find that its contribution is cancelled by the contribution of graph (b) in Figure 2. This is true for both of the temporally split bilinears. The contribution of the remaining two graphs, (a) and (c) in Figure 2, is

$$
\Delta d_{1}= \pm \frac{1}{\pi} \int d^{3} l \frac{1}{4 \Delta_{1}^{(3)}} .
$$

The upper sign is for the operator in equation (4.1) and the lower sign is for the operator in equation (4.2). Numerically, this integral has the value 19.95. For either operator we find $\Delta d_{2}=0$.

When one evaluates correlation functions of either of these operators on the lattice, a paradox appears: there is absolutely no change in the combination of gauge links and light quark propagators that is used in the temporally-split case as compared to the zero distance case. For the operator (4.2), the final gauge 
link is associated with the operator instead of the propagator, but the product is unchanged. A similar thing occurs for the operator (4.1), except that there one additional link and its inverse appear in the product and cancel. The lack of a change in the correlator is disturbing since we have found a change in the relationship of the operator to its continuum counterpart. For simplicity, in what follows we will just discuss the latter of the two temporally-split operators (4.2).

The resolution of this paradox brings us back to a subtlety already explored in the renormalization of the distance-zero operator [5]. Because the propagator used in numerical simulations of heavy quarks corresponds to an unrenormalized action, and because the heavy quark self-energy is linearly divergent, it matters whether one fits the correlation function measured in numerical simulations to $A \mathrm{e}^{-B n_{0} a}$ or to $A \mathrm{e}^{-B\left(n_{0}+1\right) a}$. As explained in reference [5], for the distance zero operator, the latter exponential is the natural one to fit to. One can repeat the argument for the temporally split distance one bilinear (4.2). In this case we find that the former exponential is the natural one to fit to.

It was also noted in reference [5] that if one uses the former exponential with the zero-distance bilinear (2.6), it can be compensated for by reducing $e$ by an amount taken from the linearly divergent part of the heavy quark self energy. ${ }^{\dagger}$ Perturbatively, the amount of the reduction was 19.95-exactly the amount by which we have found $d_{1}$ to be reduced for the operator (4.2). A similar argument can be given for the operator (4.1).

The resolution of the apparent paradox relied on a perturbative estimate for the linearly divergent part of the heavy quark self energy. The use of the operators (2.6), (4.1) and (4.2) fitted to the appropriate exponentials will only give the same results to the extent that a perturbative estimate of the linear divergence of the heavy quark self energy is accurate.

\section{Conclusions}

We have renormalized three distance-one bilinears chosen to respect the symmetries

$\dagger$ The correlator being discussed in reference [5], had both the zero-distance bilinear (2.6) and its conjugate, and the error of fitting to the former exponential was split between the two operators. If it had just been associated with one of the two operators, the error in fitting to the former exponential could have been compensated for by a reduction in $d_{1}$. 
of the lattice heavy quark action. Our results can be used whether the light quark is treated as a Wilson, staggered or naive fermion. These operators can be applied in a variety of ways. Most fundamentally, they provide a basic consistency check for this approach to determining $B$ decay matrix elements. A lattice calculation to check for agreement of the results should be undertaken.

After basic consistency is established, one can imagine three ways of applying our results for the spatially-split bilinear. This operator had a significantly smaller correction than the correction to the bilinear that is currently used for the determination of $f_{B}$ (whether the reduced or unreduced value of the heavy quark wave function renormalization is used). Hence that part of the uncertainty which is due to the choice of the value of the strong coupling is correspondingly reduced. Optimistically, one could then assume that this is the more accurately renormalized operator and simply use the resulting values. Further, one could assume that the results for both operators are accurate and fix the value of $g^{2}$ by choosing it to eliminate any disagreement between them. A third more conservative application would be to take the difference in the values for $f_{B}$, with a range of reasonable values for $g^{2}$, as an estimate of the systematic error resulting from perturbative matching and choice of discretization.

The application of the various temporally-split operators has been shown to be related to studying the dependence on extracting $f_{B}$ from lattice simulations using various exponential fitting functions. The two temporally-split operators highlight the fact that the perturbative estimate for the heavy quark self energy must be accurate or else different operators with the same naive continuum limit will give results that disagree. The values of the parameters in these fitting functions and their statistical correlation must be reported to study this dependence. This will give us another estimate of some of the systematic errors in this approach to computing $f_{B}$.

\section{Acknowledgements}

We thank Estia Eichten for discussions. OFH was supported in part by the National Science and Engineering Research Council of Canada, and les Fonds FCAR du Québec. 


\section{References}

[1] E. Eichten, in Field Theory on the Lattice, edited by A. Billoire et al., Nucl. Phys. B (Proc. Suppl.) 4 (1988) 170.

[2] G. P. Lepage and B. A. Thacker, ibid, p. 199.

[3] G. Martinelli and Y.-C. Zhang, Phys. Lett. 125B (1983) 77.

[4] P. B. Mackenzie, private communication.

[5] E. Eichten and B. Hill, Phys. Lett. B 240 (1990) 193.

[6] O. F. Hernández and B. R. Hill, Phys. Lett. B 237 (1990) 95.

[7] J. M. Flynn, O. F. Hernández and B. R. Hill, Phys. Rev. D 43 (1991) 3709.

[8] J. M. Cornwall and G. Tiktopoulos, Phys. Rev. D 15 (1977) 2937.

[9] E. Eichten and F. Feinberg, Phys. Rev. D 23 (1981) 2724.

[10] W. E. Caswell and G. P. Lepage, Phys. Lett. 167B (1986) 437.

[11] H. D. Politzer and M. B. Wise, Phys. Lett. B 208 (1988) 504.

[12] E. Eichten and B. Hill, Phys. Lett. B 234 (1990) 511.

[13] B. Grinstein, Nucl. Phys. B339 (1990) 253.

[14] H. Georgi, Phys. Lett. B 240 (1990) 447.

[15] M. J. Dugan, M. Golden, and B. Grinstein, HUTP/91-A045.

[16] Ph. Boucaud, C. L. Lin and O. Pène, Phys. Rev. D 40 (1989) 1529;

Ph. Boucaud, C. L. Lin, and O. Pène, Phys. Rev D 41 (1990) 3541(E).

[17] G. Martinelli and Y.-C. Zhang, Phys. Lett. 123B (1983) 433.

[18] G. P. Lepage and P. B. Mackenzie, in Lattice '90, ed. by U. M. Heller et al, Nucl. Phys. B (Proc. Suppl.) 20 (1991) 173.

[19] G. P. Lepage, J. Comp. Phys. 27 (1978) 192. 